\title{
Análise comparativa de duas formações vegetacionais e de seu ecótono, Miranda - MS
}

\section{Comparative analysis of two vegetation types and its ecotone, Miranda - MS}

\author{
Mariana Chaves Mota ${ }^{1}$; Pamela Cristina Santana ${ }^{2}$; Mariana $\mathrm{Hertel}^{2}$; Natalia Rizzo \\ Friol$^{2}$; Marilia Martins Cavariani²; Mario Roberto Castro Meira Filho' ${ }^{1}$; Marcelo \\ Gomes Francisco ${ }^{2}$; Jose Marcelo Domingues Torezan ${ }^{3 *}$
}

\begin{abstract}
Resumo
O Pantanal é constituído por diferentes formações vegetacionais, entre elas o Paratudal e a Mata Ciliar. Este trabalho objetivou analisar comparativamente essas duas formações, e o ecótono entre elas, quanto à diversidade e similaridade. $\mathrm{O}$ estudo foi desenvolvido na região do Passo do Lontra, às margens do rio Miranda, MS. Foram estabelecidas cinco parcelas de $10 \times 10 \mathrm{~m}$ em cada área e nelas todos os indivíduos acima de $1 \mathrm{~m}$ de altura foram contados e identificados. Foram registrados 913 indivíduos, distribuídos em 70 taxa, dos quais 60 foram identificados em nível de espécie. O índice de diversidade de Shannon (H') foi 2,715 com equidade de Pielou (J') de 0,806 no Paratudal; 3,010 e 0,835 na Mata Ciliar; e 2,739 e 0,797 no ecótono, respectivamente. Esses resultados demonstram maior diversidade na Mata Ciliar, como esperado, já que as condições do ambiente do Paratudal são mais seletivas. A similaridade florística entre o Paratudal e a Mata Ciliar, de acordo com o índice de Sorensen, foi de 0,2 o que distingue ambos os ambientes. Portanto, este trabalho evidencia a distinção entre Mata Ciliar e Paratudal, bem como os valores intermediários do ecótono.

Palavras-chave: Diversidade. Levantamento florístico. Mata ciliar. Pantanal. Paratudal. Similaridade.
\end{abstract}

\begin{abstract}
The Pantanal consists of different vegetation types, including the Paratudal, a monodominat floodable savanna, and Riparian vegetation. This study aimed to analyze diversity and similarity in samples of these two vegetations, and its ecotone. The study was developed in the region of Passo do Lontra, at the Miranda river margins. Five plots $10 \times 10 \mathrm{~m}$, were allocated in each area, where all individuals above $1 \mathrm{~m}$ height were counted and identified. Nine hundred and thirteen individuals were registered, distributed in 70 taxa, of which 60 were identified in species level. The Shannon diversity index (H') was 2.715 with Pielou Eveness (J') of 0.806 in Paratudal, 3.010 and 0.835 in Riparian vegetation, and 2.739 and 0.797 in ecotone, respectively. These results show highest diversity in the Riparian vegetation, as expected, since the Paratudal's ambient conditions are more restrictive. The floristic similarity between the Paratudal and the Riparian vegetation, according to the Sorensen index, was of 0.2 , what distinguish both environments. Therefore, this study evidences the distinction among Riparian vegetation and Paratudal, as well the existence of an intermediary values with ecotone.
\end{abstract}

Keywords: Brazilian pantanal. Diversity. Floristic survey. Paratudal. Riparian vegetation. Similarity.

\footnotetext{
1 Pós-Graduação em Ciências Biológicas, Depto. de Biologia Animal e Vegetal, Centro de Ciências Biológicas, Universidade Estadual de Londrina C.P. 6001, CEP: 86051-970 - Londrina-PR.

2 Graduação em Ciências Biológicas, Depto. de Biologia Animal e Vegetal, Centro de Ciências Biológicas, Universidade Estadual de Londrina C.P. 6001, CEP: 86051-970 - Londrina-PR.

3 Docente do Depto. de Biologia Animal e Vegetal, Centro de Ciências Biológicas, Universidade Estadual de Londrina; C.P. 6001, CEP: 86051-970 - Londrina-PR. Email: torezan@uel.br. * Autor para correspondência
} 


\section{Introdução}

O Pantanal está localizado no centro da América do Sul e sua área cobre parte do Brasil, Bolívia e Paraguai. No território brasileiro, situa-se na região Centro Oeste, inserido na bacia hidrográfica do Alto Rio Paraguai. Trata-se da maior planície da Terra sujeita a inundações periódicas, intra e inter-anual, sendo composto por vários ecossistemas (SILVA; ABDON, 1998).

Segundo Alho e Martins (1995) e Giulietti et al. (2000), o Pantanal é muito rico floristicamente, destacando-se em relação à biodiversidade devido a sua grande extensão e heterogeneidade fitofisionômica. Esta heterogeneidade tem sido relacionada às variações do relevo e topografia (PONCE; CUNHA, 1993;DAMASCENO-JUNIOR et al., 2005), à estrutura do solo (ZEILHOFER; SCHESSL, 2000) e aos padrões espaço-temporal da inundação (REBELLATO; CUNHA, 2005, ARIEIRA; CUNHA, 2006). Dentre as diversas fitofisionomias, pode-se citar as Matas Ciliares e o Paratudal (SOARES; OLIVEIRA, 2009).

A Mata Ciliar pode ser definida como toda vegetação arbórea que está localizada nas margens dos corpos d'água e desempenha funções ecológicase hidrológicas importantes em uma bacia hidrográfica (SALVADOR, 1987). Essas áreas atuam como filtros, retendo defensivos agrícolas, poluentes e sedimentos que seriam transportados para os cursos d'água, afetando diretamente a quantidade e a qualidade da água e, conseqüentemente, a fauna aquática e a população humana. Em regiões com topografia acidentada, exercem a proteção do solo contra os processos erosivos. São importantes também como corredores ecológicos, pois ligam fragmentos florestais, facilitando o deslocamento da fauna e o fluxo gênico entre as populações de espécies animais e vegetais (MARTINS, 2001).

As Matas Ciliares diferenciam-se das formações adjacentes pela estrutura, em geral, mais densa e mais alta devido principalmente à associação com o curso d'água (RIBEIRO; WALTER, 1998).
Levantamentos florísticos e fitossociológicos em remanescentes de florestas ciliares, realizados em diferentes regiões do Brasil, têm mostrado que essas áreas são muito diversas quanto à composição e estrutura fitossociológica como resultado da elevada heterogeneidade ambiental à qual estão associadas (FELFILI, 1995; SANCHEZ et al., 1999; SAMPAIO; WALTER; FELFILI, 2000; SILVA JÚNIOR, 2001).

Já o Paratudal, segundo Soares e Oliveira (2009), é uma formação savânica alagável, com estrato arbóreo com domínio de Tabebuia aurea (Bignoniaceae), o que caracteriza uma formação monodominante neste estrato. Entretanto, o estrato herbáceo apresenta alta diversidade e densidade de espécies (POTT; POTT, 1994; SILVA et al., 2000). Esse tipo de vegetação destaca-se pela intensa floração de cor amarela forte de T. aurea, aliada a caducifolia da espécie, que ocorre nos meses de agosto/setembro. Essa espécie muitas vezes cresce sobre murundus, que são montículos de terra espalhados na planície de inundação aos quais as árvores podem estar associadas. O Paratudal ocorre principalmente no sul do Pantanal até as proximidades do rio Nabileque, associado às zonas de maior influência das inundações por águas de pH alcalino, como as do Rio Miranda (SOARES; OLIVEIRA, 2009).

Entre essas duas formações vegetacionais, encontra-se o ecótono, que é a transição entre dois sistemas diferentes. Segundo Neiff (2003), o ecótono é uma área de tensão instável sem padrões próprios de variabilidade e, portanto, de diversidade, e cuja estrutura depende inteiramente das tensões que impõem duas comunidades adjacentes. A proporção de espécies (populações), a fisionomia e a diversidade no ecótono dependerão das proporções de elementos e processos gerados nos dois sistemas vegetacionais que o compõem, que por sua vez, não são sistemas isolados. A comunidade do ecótono pode conter organismos de cada uma das comunidades que se sobrepõem, além dos organismos característicos (ODUM, 1972). 
Considerando as diferenças entre essas formações vegetacionais, testou-se a hipótese de que o Paratudal e a Mata Ciliar diferem entre si, sendo a Mata Ciliar mais diversa por apresentar um ambiente menos seletivo comparado ao Paratudal, e que o ecótono apresenta parâmetros intermediários, por ser uma área de transição. Portanto, o presente estudo tem como objetivo analisar comparativamente essas formações no que se refere à diversidade e similaridade florística.

\section{Material e métodos}

Área de estudo

As áreas amostradas estão localizadas na Base de Estudos do Pantanal (BEP), pertencente à Universidade Federal do Mato Grosso do Sul, na região do Passo do Lontra, Município de Miranda, MS, nas coordenadas $19^{\circ} 34^{\prime} 37^{\prime \prime}$ S e $57^{\circ} 00^{\prime} 42^{\prime \prime} \mathrm{W}$
(Figura 1a). O clima é classificado como Aw, de acordo com a classificação climática de Köppen, e é caracterizado por clima tropical de savana, com uma estação seca pronunciada e com precipitação média anual entre 1000 e $1200 \mathrm{~mm}$ (SALOMÃO et al., 2008). Na região, o período mais intenso de chuvas ocorre de novembro a março, ocasionando um período de inundação com início em dezembro e pico máximo em fevereiro. Entre os meses de setembro a novembro, ocorre déficit hídrico (BRASIL, 1980). O período mais frio ocorre de maio a agosto, com média mensal mínima de $20^{\circ} \mathrm{C}$, e o período mais quente ocorre de novembro a fevereiro, com média máxima em torno de $27^{\circ} \mathrm{C}$.

As áreas de amostragem estão localizadas em duas formações vegetacionais (Paratudal e Mata Ciliar), e na área de transição entre estas (ecótono) (Figura 1b).

Figura 1. Área de Estudo. (a) Área em cinza representa a região do Pantanal Sul Mato-grossense. BEP Base de Estudos do Pantanal, região do Passo do Lontra, Miranda, MS. Fonte: Pereira et al. (2007). (b) Localização dos ambientes amostrados: Mata ciliar, Ecótono e Paratudal. Fonte: Google Earth (2010).

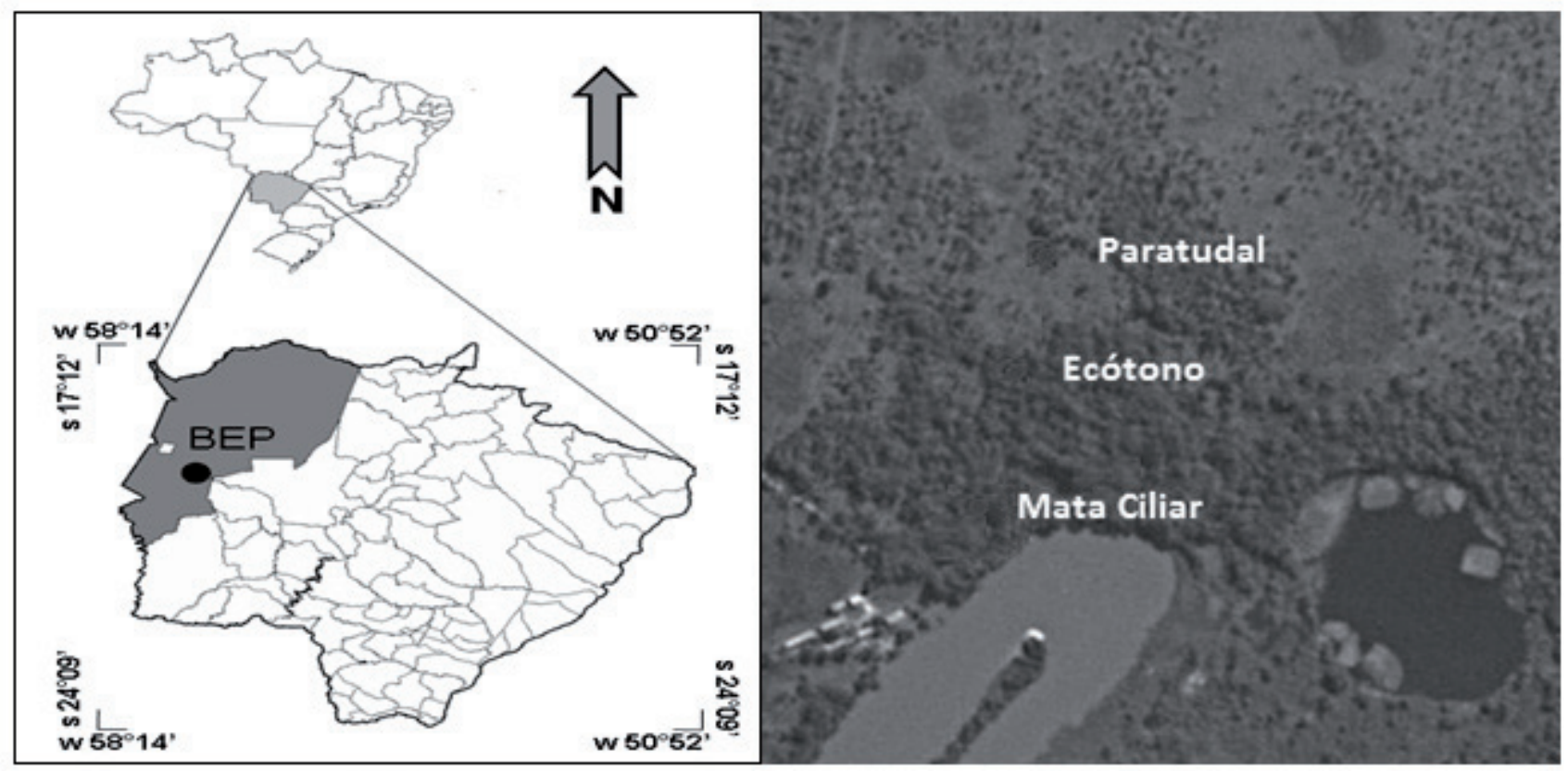

(a)

(b) 


\section{Coleta de dados}

As coletas foram realizadas entre os dias $23 \mathrm{e}$ 27 de agosto de 2010. Em cada uma das três áreas foram delimitadas 5 parcelas de $10 \times 10 \mathrm{~m}\left(100 \mathrm{~m}^{2}\right)$ cada, totalizando 15 parcelas e $1500 \mathrm{~m}^{2}$ de área amostrada. Foram coletadas amostras da estrutura vegetativa, e, quando possível, da reprodutiva, de todos os indivíduos com altura igual ou superior a $1 \mathrm{~m}$, não ocorrendo distinção dos hábitos dos indivíduos. O material coletado foi armazenado em sacos plásticos e transportado para o laboratório para posterior herborização. A identificação taxonômica foi efetuada mediante consulta a especialistas ou por meio de literatura especializada. A sinonímia, a grafia e a abreviação dos nomes dos autores dos taxa foram atualizadas, mediante consulta ao índice de espécies do banco de dados do Missouri Botanical Garden (MISSOURI BOTANICAL GARDEN, 2010). O sistema de classificação adotado foi o APG II (2003).

\section{Análise dos dados}

Para avaliar a diversidade florística da área utilizou-se o índice de Shannon (H') e a eqüidade de Pielou (J') a ele correspondente (BROWER; ZAR, 1984). Para verificar se os valores de H' eram significativos, utilizou-se a estatística $t$ (t de Hutcheson $\mathrm{p}<0,05)$ (ZAR, 1996). Para a análise de similaridade entre as áreas, foi utilizado o Índice de Sørensen (MUELLER-DOMBOIS; ELLENBERG, 1974).

\section{Resultados e discussão}

Foram registrados 913 indivíduos, distribuídos em 70 taxa, dos quais 60 foram identificados em nível de espécie $(85,7 \%)$, seis em nível de gênero $(8,6 \%)$, dois em nível de família $(2,8 \%)$ e dois permaneceram como morfoespécies sem identificação taxonômica $(2,8 \%)$ (Tabela 1$)$.

No Paratudal, foram identificados 380 indivíduos (tabela 1), com predominância de Sebastiania hispida, Lippia alba, Paullinia pinnata e Sapium haematospermum. Soares e Oliveira (2009), em estudo realizado no Paratudal do Pantanal de Miranda, a partir de amostragens de indivíduos com diâmetro a altura do peito (DAP) $\geq$ a $5 \mathrm{~cm}$, encontraram predominância de Tabebuia aurea, Inga edulis, Erythroxylum anguifugum e Tabebuia heptaphylla. Esta diferença entre as espécies predominantes pode ser resultado do método utilizado para inclusão dos indivíduos. O número expressivo de T. heptaphylla, (Piúva-do-pantanal) encontrado no local é explicado por Pott e Pott (1994), que indicaram ser essa espécie muito comum em todo Pantanal e estar associada a rios e canais de drenagem, formando muitas vezes o chamado Piuval às margens desses locais.

Na Mata Ciliar foram registrados 209 indivíduos (tabela 1), cujas espécies mais abundantes foram Bactris glaucescens, Eugenia florida, Genipa americana. Salomão et al. (2008), em levantamento florístico, por meio do método de quadrantes, em matas ciliares do Rio Miranda, encontraram Inga vera, Tabebuia heptaphylla, Ocotea diospiryfolia e Neea hermaphrodita com maior número de indivíduos.

No ecótono, foram registrados 324 indivíduos (tabela 1), sendo Sebastiania hispida, Psychotria cartagoensis e Smilax sp. as espécies com maior número de indivíduos.

A similaridade florística entre Paratudal e Mata Ciliar, baseada no índice de Sørensen, foi de 0,20, valor considerado baixo (IS $<0,50$ ) para duas formações vegetacionais (KENT; COKER, 1994). Os baixos valores registrados por este índice corroboram os estudos que indicam a grande heterogeneidade florística encontrada em áreas de cerrado (OLIVEIRA-FILHO; RATTER, 2002), geralmente distribuído em mosaicos (RATTER; BRIDGEWATER; RIBEIRO, 2003). As espécies que ocorreram em ambas as áreas foram Erythroxylum anguifugum, Neea hermaphrodita, Paullinia pinnata, Tabebuia heptaphylla, Eugenia egensis, Vitex cymosa e a Morfoespécie 2. 
Tabela 1 - Listagem florística de famílias, gêneros e espécies com respectivos números de indivíduos registrados em áreas de Paratudal, ecótono e Mata Ciliar, Rio Miranda, Município de Miranda, MS.

\begin{tabular}{|c|c|c|c|}
\hline \multirow{2}{*}{\multicolumn{4}{|c|}{$\begin{array}{l}\text { Família/Espécie } \\
\text { AMARANTHACEAE }\end{array}$}} \\
\hline & & & \\
\hline Pfaffia glomerata (Spreng.) Pedersen & 4 & 0 & 0 \\
\hline $\begin{array}{l}\text { Rollinia sylvatica (A. St.-Hil.) Martius } \\
\text { APOCYNACEAE }\end{array}$ & 0 & 1 & 0 \\
\hline Schubertia grandiflora Mart. \& Zucc. & 5 & 2 & 0 \\
\hline $\begin{array}{l}\text { Thevetia bicornuta Müll. Arg. } \\
\text { ARECACEAE }\end{array}$ & 11 & 1 & 0 \\
\hline Bactris glaucescens Drude & 0 & 0 & 37 \\
\hline Copernicia alba Morong ex Morong \& Britton & 0 & 0 & 1 \\
\hline Desmoncus cuyabaensis Barb. Rodr. & 0 & 0 & 2 \\
\hline $\begin{array}{l}\text { Scheelea phalerata (Mart. ex Spreng.) Burret } \\
\text { ASTERACEAE }\end{array}$ & 0 & 0 & 1 \\
\hline $\begin{array}{l}\text { Eupatorium odoratum L. } \\
\text { ARISTOLOCHIACEAE }\end{array}$ & 1 & 0 & 0 \\
\hline $\begin{array}{l}\text { Aristolochia ridicula N.E. Br. } \\
\text { BIGNONIACEAE }\end{array}$ & 0 & 3 & 0 \\
\hline Tabebuia aurea (Silva Manso) Benth. \& Hook. f. ex S. Moore & 21 & 4 & 0 \\
\hline $\begin{array}{l}\text { Tabebuia heptaphylla (Vell.) Toledo } \\
\text { CALOPHYLLACEAE }\end{array}$ & 2 & 5 & 7 \\
\hline $\begin{array}{l}\text { Calophyllum brasiliense Cambess. } \\
\text { CANNABACEAE }\end{array}$ & 0 & 0 & 1 \\
\hline $\begin{array}{l}\text { Celtis pubescens Spreng. } \\
\text { CELASTRACEAE }\end{array}$ & 0 & 0 & 1 \\
\hline $\begin{array}{l}\text { Salacia elliptica (Mart. ex Schult.) G. Don } \\
\text { CHRYSOBALANACEAE }\end{array}$ & 0 & 0 & 3 \\
\hline Couepia uiti (Mart. \& Zucc.) Benth. ex Hook. f. & 0 & 0 & 1 \\
\hline Hirtella glandulosa Spreng. & 0 & 0 & 1 \\
\hline Licania parviflora Benth. & 1 & 0 & 0 \\
\hline $\begin{array}{l}\text { Licania sp. } \\
\text { CONVOLVULACEAE }\end{array}$ & 0 & 0 & 2 \\
\hline $\begin{array}{l}\text { Ipomoea } \text { sp. } \\
\text { ERYTHROXYLACEAE }\end{array}$ & 18 & 0 & 0 \\
\hline $\begin{array}{l}\text { Erythroxylum anguifugum Mart. } \\
\text { EUPHORBIACEAE }\end{array}$ & 12 & 11 & 3 \\
\hline Sapium haematospermum Müll. Arg. & 22 & 3 & 0 \\
\hline Sapium hasslerianum Huber & 5 & 1 & 0 \\
\hline Sapium longifolium (Müll. Arg.) Huber & 4 & 1 & 0 \\
\hline $\begin{array}{l}\text { Sebastiana hispida Pax } \\
\text { FABACEAE }\end{array}$ & 98 & 49 & 0 \\
\hline Albizia polyantha (A. Spreng.) G.P. Lewis & 0 & 0 & 6 \\
\hline Andira cujabensis Benth. & 0 & 0 & 3 \\
\hline Cassia grandis L. f. & 0 & 0 & 2 \\
\hline Senna pendula (Humb. \& Bonpl. ex Willd.) H.S. Irwin \& Barneby & 3 & 0 & 0 \\
\hline Senna sp. & 6 & 0 & 0 \\
\hline
\end{tabular}


Continuação Tabela 1

Zygia inaequalis (Humb. \& Bonpl. ex Willd.) Pittier

LAMIACEAE

Hyptis sp.

Vitex cymosa Bertero ex Spreng.

0

1

LAURACEAE

Ocotea suaveolens (Meisn.) Benth. \& Hook. f. ex Hieron.

Lauraceae 1

LOGANIACEAE

Strychnos pseudoquina A. St.-Hil.

MALPIGHIACEAE

Byrsonima orbignyana A. Juss.

Heteropterys sp.

MALVACEAE

Guazuma ulmifolia Lam.

Sida cerradoensis Krap.

MELIACEAE

Trichilia catigua A. Juss.

Trichilia sp.

MENISPERMACEAE

Cissampelus pareira $\mathrm{L}$.

MYRTACEAE

Campomanesia sessiliflora (O. Berg) Mattos

Eugenia egensis DC.

Eugenia florida DC.

Gomidesia palustris (DC.) D. Legrand

Psidium guajava L.

NYCTAGINACEAE

Neea hermaphrodita S. Moore

POLYGONACEAE

Coccoloba ochreolata Wedd.

Triplaris americana L.

RUBIACEAE

Alibertia sessilis (Vell.) K. Schum.

Genipa americana L.

Psychotria cartagoensis Nepokroeff

Randia armata (Sw.) DC.

Tocoyena formosa (Cham. \& Schltdl.) K. Schum.

RUTACEAE

Esenbeckia sp.

Fagara hassleriana Chodat

SALICACEAE 1

SAPINDACEAE

Paullinia elegans Cambess.

Paullinia pinnata L.

SMILACACEAE

$\begin{array}{lll}0 & 11 & 6 \\ 0 & 2 & 0 \\ 0 & 0 & 2\end{array}$

$\begin{array}{lll}8 & 14 & 0 \\ 0 & 0 & 15 \\ 0 & 0 & 2 \\ 16 & 0 & 0\end{array}$

$\begin{array}{lll}0 & 0 & 1 \\ 0 & 0\end{array}$

$\begin{array}{lll}0 & 0 & 1\end{array}$

$\begin{array}{lll}0 & 6 & 1\end{array}$

100

$\begin{array}{lll}1 & 3 & 8\end{array}$

$\begin{array}{lll}0 & 0 & 20\end{array}$

$\begin{array}{lll}0 & 0 & 8\end{array}$

$\begin{array}{lll}0 & 1 & 0\end{array}$

$\begin{array}{lll}1 & 2 & 6\end{array}$

$\begin{array}{lll}0 & 0 & 4\end{array}$

$\begin{array}{lll}0 & 1 & 0\end{array}$

$\begin{array}{lll}0 & 0 & 1\end{array}$

$\begin{array}{lll}0 & 3 & 19\end{array}$

$\begin{array}{lll}0 & 45 & 13\end{array}$

$0 \quad 0 \quad 1$

$0 \quad 0 \quad 1$

$0 \quad 0 \quad 1$

$\begin{array}{lll}0 & 21 & 0\end{array}$

$0 \quad 1 \quad 0$

$0 \quad 0 \quad 2$

$\begin{array}{lll}22 & 27 & 9\end{array}$


Smilax sp.

\section{URTICACEAE}

Cecropia pachystachya Trécul

VERBENACEAE

Lippia alba (Mill.) N.E. Br. ex Britton \& P. Wilson

Morfoespécie 1

Morfoespécie 2
1

0

33

20

8
32

0

31

2

0
0

4

0

0
Em relação ao levantamento florístico do ecótono, foram encontradas 17 espécies em comum ao Paratudal, sendo o índice de similaridade de Sørensen de 0,90. Já para mata ciliar, foram encontradas 11 espécies em comum, com índice de Sørensen de 0,30, dado que revela a maior similaridade do ecótono com o Paratudal (tabela 2).

O ecótono é definido como uma região de transição entre dois ou mais ecossistemas, produzida por invasão mútua (ODUM, 1972), portanto as amostragens em ecótonos são arbitrárias. A escolha da localização das parcelas é aleatória, o que pode ocasionar o favorecimento amostral numa faixa de transição mais próxima de uma das áreas do que da outra. Isso pode justificar os valores de similaridade encontrados para ecótono/Paratudal e ecótono/Mata ciliar. Além disso, os incêndios provocados pelo manejo do Paratudal como pastagem no passado recente pode ter contribuído para uma "savanização" do ecótono.

Tabela 2 - Índice de Similaridade de Sørensen entre Paratudal, Mata Ciliar e ecótono, Rio Miranda, Município de Miranda, MS.

\begin{tabular}{lccc}
\hline & Paratudal & Mata ciliar & Ecótono \\
\hline Paratudal & 1 & 0,2 & 0,9 \\
Mata Ciliar & - & 1 & 0,3 \\
Ecótono & - & - & 1 \\
\hline
\end{tabular}

A diversidade florística encontrada no Paratudal, segundo o índice de Shannon (H'), foi de 2,715, com eqüidade (J') de 0,806 . Já a diversidade florística encontrada para a Mata Ciliar foi de 3,010, com eqüidade (J') de 0,835 , valor comparativamente diferente ao encontrado por Salomão et al. (2008), cuja diversidade foi de 2,770 para um trecho de Mata Ciliar do Rio Miranda. Essa diferença entre os valores de diversidade encontrados pode ser justificada devido à utilização de diferentes métodos de amostragem, sendo que Salomão et al. (2008) utilizaram a metodologia de quadrantes ou point-centered quarter method, baseado em Rodrigues (1988). A comparação realizada entre o H' do Paratudal e o da Mata Ciliar, pelo teste de t de
Hutcheson demonstrou que há diferença significativa entre estas áreas $(p=0,0004)$, evidenciando que a Mata Ciliar apresenta maior diversidade, quando comparada ao Paratudal.

Este resultado era esperado, tendo em vista que as Matas Ciliares são fortemente influenciadas por uma série de fatores físicos locais, como as variações edáficas e topográficas. A associação das matas ciliares com as formações adjacentes resulta numa composição florística mista, com espécies típicas das margens de rios e de outras formações adjacentes (IVANAUSKAS; RODRIGUES; NAVE, 1997). Porém, como a Mata Ciliar está associada a um curso d'água, ela se apresenta, em geral, mais densa e mais alta (RIBEIRO; WALTER, 
2001). Estudos realizados na região do Pantanal têm demonstrado elevada diversidade quanto à sua composição florística (SAMPAIO; WALTER; FELFILI, 2000; SILVA JÚNIOR, 2001).

A menor diversidade encontrada no Paratudal ocorre devido à grande seletividade do solo, se levado em consideração o nível de cálcio e magnésio (SOARES; OLIVEIRA, 2009; PRANCE; SCHALLER, 1982). Durante o período de inundação, além da possibilidade de morte decorrente da submersão de indivíduos jovens, o $\mathrm{pH}$ do solo é alterado, dificultando a germinação e instalação das plântulas. Outro fator limitante, consequente da inundação, é a hipoxia, caracterizada pela diminuição no teor de oxigênio $\left(\mathrm{O}_{2}\right)$, visto que o $\mathrm{O}_{2}$ dissolvido na água é rapidamente consumido pela ação de microrganismos, mesofauna e raízes, resultando no acúmulo de gases tóxicos como $\mathrm{CO}_{2}, \mathrm{~N}_{2}, \mathrm{H}_{2}, \mathrm{CH}_{4}, \mathrm{H}_{2} \mathrm{~S}$ (PEZESHKI, 1994), o que pode provocar desordens metabólicas na planta, diminuindo a eficiência na utilização de carbono e aumentando a produção de etanol e lactato (MARSCHNER, 1986). Apesar de alguns autores afirmarem que os murundus não são submersos pelas águas, isso pode acontecer, como indicam as marcas nos troncos, ocasionadas pela água, bem acima dos montículos de terra, evidenciando o nível de inundação atingido.

Outro importante fator a ser considerado é a ocorrência periódica do fogo no Paratudal, que atua selecionando somente as espécies resistentes a esse fator. Nessa fitofisionomia, a cobertura vegetal responsável pelo fornecimento do material combustível para queima é caracterizada, principalmente, por um acúmulo natural de biomassa de gramíneas não pastadas. Além disso, em vegetações ralas ou abertas, como o Paratudal, o ar circula com facilidade, diminuindo a umidade do ar, contribuindo para acelerar a secagem do material combustível, o que permite que o fogo se propague com maior facilidade (OLIVEIRA et al., 2002). Entre os efeitos adversos do fogo está a diminuição da diversidade de espécies, que é agravada com o aumento da freqüência e intensidade das queimadas (SAMBUICHI, 1991). Já uma floresta densa, como a Mata Ciliar, proporciona um microclima mais ameno, consequentemente, os combustíveis apresentam um conteúdo de umidade maior e mais estável do que uma floresta rala ou aberta, o que dificulta a ocorrência de incêndios (OLIVEIRA et al., 2002).

Em relação ao ecótono, a diversidade florística encontrada foi de 2,739 (H') com eqüidade (J') de 0,7975, valores intermediários aos encontrados para Paratudal e Mata Ciliar. Estes valores eram esperados, tendo em vista que o ecótono, sendo uma área de transição, deve apresentar valores intermediários para os parâmetros que caracterizam a estrutura de cada ambiente (NEIFF, 2003).

Pode-se concluir que o Paratudal e a Mata Ciliar possuem baixa similaridade florística, ou seja, estas duas formações vegetacionais diferem entre si, evidenciando a heterogeneidade florística presente no Pantanal. Em relação à diversidade, essas áreas também diferem entre si, sendo que o maior índice de diversidade foi encontrado para a Mata Ciliar. Esse resultado era esperado, pois o Paratudal é um ambiente que apresenta fatores limitantes para o estabelecimento de diversas espécies vegetais, dentre eles a seletividade edáfica, a ação do fogo e as alterações no pH do solo e hipoxia, ocasionadas por inundações periódicas. Quanto ao ecótono os valores de diversidade e similaridade foram intermediários aos encontrados para Paratudal e Mata Ciliar, valores que eram esperados, porque o ecótono é uma área de transição.

\section{Agradecimentos}

Aos docentes da disciplina de Biologia de Campo pelo apoio e orientação, aos colegas de curso e a Edson Mendes Francisco pelo apoio na coleta dos dados, Dr. Edmilson Bianchini e Valéria Teodoro pelas críticas e sugestões, e aos funcionários da Base de Estudos do Pantanal (BEP) da UFMS e ao Departamento de Biologia Animal e Vegetal da 
UEL pelo apoio logístico.

\section{Referências}

ALHO, C. J. R.; MARTINS, E. S. De grão em grão, o cerrado perde espaço (cerrado-impactos do processo de ocupação). Brasília: WWF, 1995. p. 205-254.

APG II (ANGIOSPERM PHYLOGENY GROUP II). An update of the Angiosperm Phylogeny Group classification for the orders and families of flowering plants: APGII. Botanical Journal of the Linnean Society, v. 141, p. 399436, 2003.

ARIEIRA, J.; CUNHA, N. C. Fitossociologia de uma floresta inundável monodominante de Vochysia divergens Pohl (Vochysiaceae), no Pantanal Norte, MT, Brasil. Acta Botanica Brasilica, São Paulo, v. 20, n. 3, p. 569-580, 2006.

BRASIL. Ministério de Minas de Energia. Departamento Nacional da produção mineral. Projeto RADAM Brasil. Folhas sc. 21. Juremo: geomorfologia, pedologias, vegetação e uso potencial da terra, Rio de Janeiro: 1980. (Levantamento de Recursos Naturais, v. 20).

BROWER, J. E.; ZAR, J. H. Field and laboratory methods for general ecology. Boston: W.C. Brown Publishers, 1984.

DAMASCENO-JUNIOR, G. A.; SEMIR, J.; SANTOS, F. A. M.; LEITÃO-FILHO, H. F. Structure, distribution of species and inundation in a riparian forest of Rio Paraguai, Pantanal, Brazil. Flora, Jena, v. 200, n. 2, p. 119-135, 2005.

FELFILI, J. M. Diversity, structure and dynamics of a gallery forest in central Brazil. Vegetatio, The Hague, v. 117, p. 1-15, 1995.

GIULIETTI, A. M.; HARLEY, R. M.; QUEIROZ, L. P.; WANDERLEY, M. G. L.; PIRANI, J. R. Caracterização e endemismos nos campos rupestres da Cadeia do Espinhaço. In: CAVALCANTI, T. B.; WALTER, B. M. T. (Org.). Tópicos atuais em botânica. Brasília: SBB/ Embrapa, 2000. p. 311-318.

IVANAUSKAS, N. M.; RODRIGUES, R. R.; NAVE, A. G. Aspectos ecológicos de um trecho de floresta de brejo em Ipatinga, SP: florística, fitossociologia e seletividade de espécies. Revista Brasileira de Botânica, São Paulo, v. 20, n. 2, p. 139-153, 1997.

KENT, M.; COKER, P. Vegetation description and analysis. New York: John Wiley \& Sons, 1994.

MARSCHNER, H. Mineral nutrition of higer plants. London: Academic Press, 1986.
MARTINS, S. V. Recuperação de matas ciliares. Viçosa: Aprenda Fácil, 2001.

MISSOURI BOTANICAL GARDEN. Banco de dados. Disponível em: <http://www.mobot.org/mobot/research/ alldb.shtml>. Acesso em: 10 out. 2010.

MUELLER-DOMBOIS, D.; ELLENBERG, H. Aims and methods for vegetation ecology. New York: John Wiley \& Sons, 1974.

NEIFF, J. J. Planícies de inundação são ecótonos? In: HENRY, R. (Org.). Ecótonos nas interfaces dos ecossistemas aquáticos. São Carlos: Rima, 2003. p. 2946.

ODUM, E. P. Ecologia. 3. ed. Rio de Janeiro: Guanabara Koogan, 1972.

OLIVEIRA FILHO, A. T.; RATTER, J. A. Vegetation physiognomies and woody flora of the cerrado biome. In: OLIVEIRA, P. S. M. C.; MARQUIS, R. J. (Ed.). The cerrados of Brazil: ecology and natural history of a Neotropical savanna. New York: Columbia University Press, 2002. p. 91-120.

OLIVEIRA, D. S.; BATISTA, A. C.; SOARES, R. V.; SLUTTER, C. R. Fire risk mapping for pine and eucalyptus stands in Três Barras, State of Santa Catarina, Brazil. In: INTERNATIONAL CONGRESS OF FOREST FIRE RESEARCH, 4., 2002, Coimbra. Anais... Coimbra: Millpress, 2002. p. 1-12.

PEREIRA, G.; MORAES, E. C.; ARAI, E.; OLIVEIRA, L. G. L. Estudo preliminar da estimativa da reflectância e albedo de microssistemas pantaneiros a partir de imagens de satélite. Revista Brasileira de Cartografia, Rio de Janeiro, v.59, p.55-61, 2007.

PEZESHKI, S. R. Plant responses to flooding. In: WILKINSON, R. E. (Ed.). Plant environment interactions. New York: Dekker, 1994. p p.289-321.

PONCE, V. M.; CUNHA, C. N. Vegetated eartmounds in tropical savannas of Central Brazil: a synthesis With special reference to the Pantanal do Mato Grosso. Journal of Biogeography, Oxford, v. 20, p. 219-225, 1993.

POTT, V. J.; POTT, A. Plantas do Pantanal. Corumbá: Embrapa/Pantanal, 1994.

PRANCE, G. T.; SCHALLER, G. B. Preliminary study of some vegetation types of the Pantanal, Mato Grosso, Brazil. Brittonia, Bronx, v. 34, n. 2, p. 228-251, 1982.

RATTER, J. A.; BRIDGEWATER, S.; RIBEIRO, J. F. Analysis of the floristic composition of the Brazilian cerrado vegetation III: comparison of the woody vegetation of 376 areas. Edinburgh Journal of Botany, Edinburgh, v. 60, p. 57-109, 2003. 
REBEllato, L.; CUNHA, C. N. Efeito do "fluxo sazonal mínimo da inundação" sobre a composição e estrutura de um campo inundável no Pantanal de Poconé MT, Brasil. Acta Botanica Brasilica, Porto Alegre, v. 19, n. 4, p. 789-799, 2005.

RIBEIRO, J. F.; WALTER, B. M. T. As matas de galeria no contexto do bioma cerrado. In: RIBEIRO, J. F. (Ed.). Cerrado: caracterização e recuperação de matas de galeria. Planaltina: EMBRAPA/Cerrados, 2001. p. 29-47.

Fitofisionomias do Bioma Cerrado. In: SANO, S. M.; ALMEIDA, S. P. (Org.). Cerrado: ambiente e flora. Planaltina: EMBRAPA/Cerrados, 1998. p. 89-166.

RODRIGUES, R. R. Métodos fitossociológicos mais usados. Campinas: CATI, 1988.

SALOMÃO, A. K. D.; PONTARA, V.; SELEME, E. P.; BUENO, M. L.; FAVA, W. S.; DAMASCENO JÚNIOR, G. A.; POTT, A. Fitossociologia e florística de um trecho de mata ciliar do Rio Miranda, Miranda, MS, Brasil. In: SIMPÓSIO NACIONAL DO CERRADO, 9., 2008, Brasília. Anais... Brasília: Embrapa Cerrados, 2008. p. 1-7.

SALVADOR, J. L. G. Considerações sobre as matas ciliares e a implantação de reflorestamentos mistos nas margens de rios e reservatórios. CESP Série Divulgação e Informação, São Paulo, n. 105, p. 1-29, 1987.

SAMBUICHI, R. H. R. Efeitos de longo prazo do fogo periódico sobre a fitossociologia da camada lenhosa de um cerrado em Brasília, DF. 1991. Dissertação (Mestrado em Ecologia) - Universidade de Brasília, Brasília.

SAMPAIO, A. B.; WALTER, B. M. T.; FELFILI, J. M. Diversidade e distribuição de espécies arbóreas em duas matas de galeria na micro-bacia do Riacho Fundo, Distrito Federal. Acta Botanica Brasilica, São Paulo, v. 14, n. 2, p. 197-214, 2000.

SANCHEZ, M.; PEDRONI, F.; LEITÃO-FILHO, H. F.; CESAR, O. Composição florística de um trecho de floresta ripária na Mata Atlântica em Picinguaba, Ubatuba, SP. Revista Brasileira de Botânica, São Paulo, v. 22, n. 1, p. 3-42, 1999.

SILVA JÚNIOR, M. C. Comparação entre matas de galeria no Distrito Federal e a efetividade do código florestal na proteção de sua diversidade arbórea. Acta Botanica Brasilica, São Paulo, v. 15, n. 1, p. 139-146, 2001.

SILVA, J. S. V.; ABDON, M. M. Delimitação do Pantanal brasileiro e suas sub-regiões. Pesquisa Agropecuária Brasileira, Brasília, v. 33, p. 1703-1711, 1998.

SILVA, M. P.; MAURO, R.; MOURÃO, G. M.; COUTINHO, M. Distribuição e quantificação de classes de vegetação do Pantanal através de levantamento aéreo. Revista Brasileira de Botânica, São Paulo, v. 23, n. 2, p. 143-152, 2000.

SOARES, J. J.; OLIVEIRA, A. K. M. O paratudal do pantanal de Miranda, Corumbá-MS, Brasil. Revista Árvore, Viçosa, v. 33, n. 2, p. 339-347, 2009.

ZAR, J. Biostatistical analysis. 3. ed. New Jersey: Prentice Hall, 1996.

ZEILHOFER, P.; SCHESSL, M. Relationship between vegetation and environmental conditions in the Northern Pantanal of Mato Grosso, Brazil. Journal of Biogeography, Oxford, v. 27, n. 1, p. 159-168, 2000.

Recebido em 17 de maio de 2011 - Received on May 17, 2011

Aceito em 18 de julho de 2011 - Accepted on July 18, 2011 